\title{
Depinning of a superfluid vortex line by Kelvin waves
}

\author{
L.A.K. Donev, L. Hough, and R.J. Zieve \\ Physics Department, University of California at Davis
}

\begin{abstract}
We measure the interaction of a single superfluid vortex with surface irregularities. While vortex pinning in superconductors usually becomes weaker at higher temperatures, we find the opposite behavior. The pinning steadily increases throughout our measurement range, from $0.15 T_{c}$ to over $0.5 T_{c}$. We also find that moving the other end of the vortex decreases the pinning, so we propose Kelvin waves along the vortex as a depinning mechanism.
\end{abstract}

PACS numbers: $67.40 . \mathrm{Vs}$, 74.60.Ge

Pinning sites can trap vortices in a variety of systems. In superconductors, where vortex motion leads to nonzero resistance, a vast amount of work has been devoted to preventing such motion by introducing defects. Experimental work has shown that defects comparable in size to the vortex core make effective pin centers, and that straight [1] or splayed [2] line defects can increase the pin strength. Yet the mechanisms by which vortices interact with pin sites remain unresolved. Experimentally, pinning in superconductors becomes weaker as temperature increases, in accord with the general assumption that depinning occurs through thermal activation over local energy barriers. In some materials, a constant rate of vortex motion at low temperature indicates a crossover to quantum tunneling between pin sites [3]. No experiments have been able to probe the actual interactions. Similar issues appear in studies of neutron stars [ $₫$ ], where vortex pinning or friction could account for angular momentum irregularities [5].

Here we report studies of a single vortex line in superfluid helium. We observe a steady increase of pinning as temperature increases, for $0.15<T / T_{c}<0.6$, and suggest that this unusual temperature dependence comes from the interaction of the pin site with oscillations along the vortex line. Studying one vortex rather than a sizeable collection simplifies some of the issues in pinning. Helium is also unusual in that the pinning occurs only at the end of the vortex, so our vortex interacts with a single pin site. Surface pinning dominates in superconductors as well for very clean samples [6, 7] or those with surface structures such as magnetic dots [8].

Our measurements use a brass cylinder, $50 \mathrm{~mm}$ long and filled with liquid helium. A fine superconducting wire, about $12 \mu \mathrm{m}$ diameter, is stretched along the cell, displaced from the cylinder's axis by about $0.8 \mathrm{~mm}$. The data reported here come from two cells, one drilled with inner diameter $2.9 \mathrm{~mm}$ (cell A) and the other reamed to diameter $3.1 \mathrm{~mm}$ (cell B). The walls of cell B appear smoother. A pumped ${ }^{3} \mathrm{He}$ cryostat can cool the cell to $300 \mathrm{mK}$. We apply a $250 \mathrm{G}$ field perpendicular to the wire. A current pulse of a few milliamperes through the wire excites the wire's vibration by a Lorentz force. The ensuing motion in the magnetic field induces an emf across the wire, which we amplify and digitize.

In the presence of fluid circulation, vorticity can become trapped on the wire. Trapped circulation alters the wire's vibrational normal modes and splits the fundamental frequency [9, 10], causing the plane of vibration to precess. A vortex can extend along the entire wire or along only a portion. In the latter case, shown in the inset of Figure 1, the vortex core leaves the wire and traverses the fluid as a free vortex [11, 12]. The splitting of the wire's normal modes due to a partially attached vortex increases with the length of the vortex on the wire. We fit the oscillation envelope to an exponentially damped sine wave to extract the beat frequency. We then convert the beat frequency to an effective circulation, that is, the constant circulation along the wire that would produce that beat frequency. For a partial vortex we can convert the beat frequency to the location of the point where the free vortex meets the wire.

For the experiments discussed here, we rotate the cryostat to create circulation but make our measurements, on the behavior of a partially trapped vortex line, with the cryostat stationary. The free end of the vortex precesses around the wire, driven by the flow field of the trapped portion. Since the wire

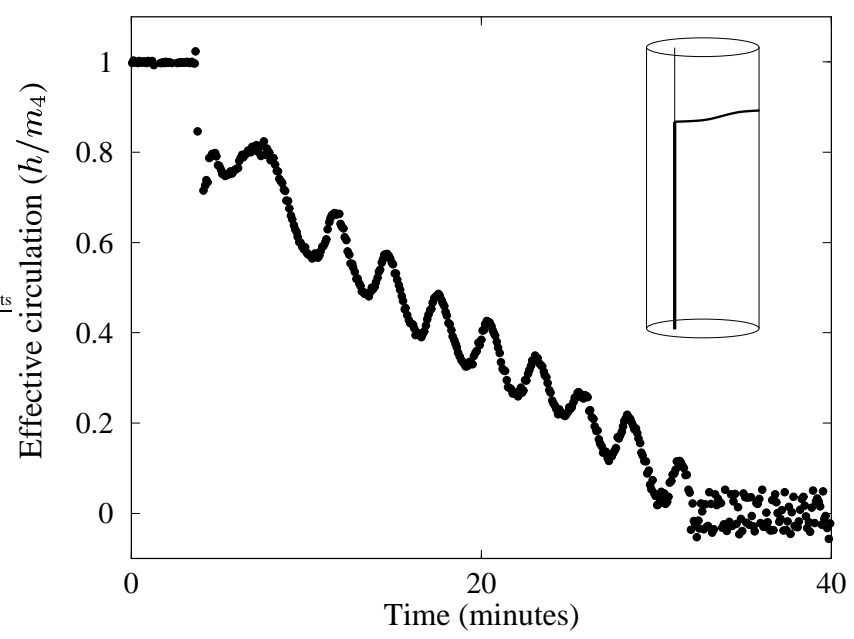

FIG. 1: Unwinding of quantized vortex from wire at $330 \mathrm{mK}$ in cell A. The inset shows a schematic of the vortex and cell. 
is off-center, the point where the free vortex attaches to the wire moves up and down during the precession to conserve energy. We detect this motion from the oscillations it produces in the beat frequency [11, 12]. An example is shown in Figure 11. The oscillation is superimposed on a steady shortening of the trapped vorticity since the free vortex line dissipates energy as it moves. These oscillations occur only at intermediate circulation, stopping when the vortex is completely on or off the wire.

As both experiments [13] and computations [14 have shown, wall roughness can pin vortices. A bump on the wall distorts the velocity field so as to pull the end of the vortex onto the bump. The vortex may settle into a metastable pinned state, with its shape determined by the local fluid velocity, as in the Figure 2 diagram. A larger velocity field may bend the vortex so far to one side that it moves off the bump entirely. The critical velocity for working free depends on the size of the bump [14, 15]. Figure 2 shows a pinning event in our cell. The peak at the left, associated with precession, suddenly gives way to faster, smaller oscillations around a constant level. The rapid oscillation has period $33 \mathrm{~s}$ and corresponds to the lowest Kelvin mode, a transverse wave on the free vortex [16]. On an infinite straight vortex, the Kelvin mode with quarter-wavelength $0.15 \mathrm{~cm}$ has period 46 seconds, but the cell geometry alters the period [15]. Both the Kelvin wave along the vortex and the resulting attachment point motion are clearly visible in our computer simulations of pinning events, and in other simulations of depinning [17].

A recent experiment found evidence for single nanometerscale vortices pinning and depinning in a small orifice [18]. The critical flow velocity required to depin the vortex showed no temperature dependence, which was taken as evidence of quantum tunneling [18]. In our experiment, the vortex frequently precesses without pinning, suggesting that the flow velocity in the cell is close to the critical velocity for depinning. Near this critical velocity, the energy barrier to depinning is small, so one might expect depinning to be easier at

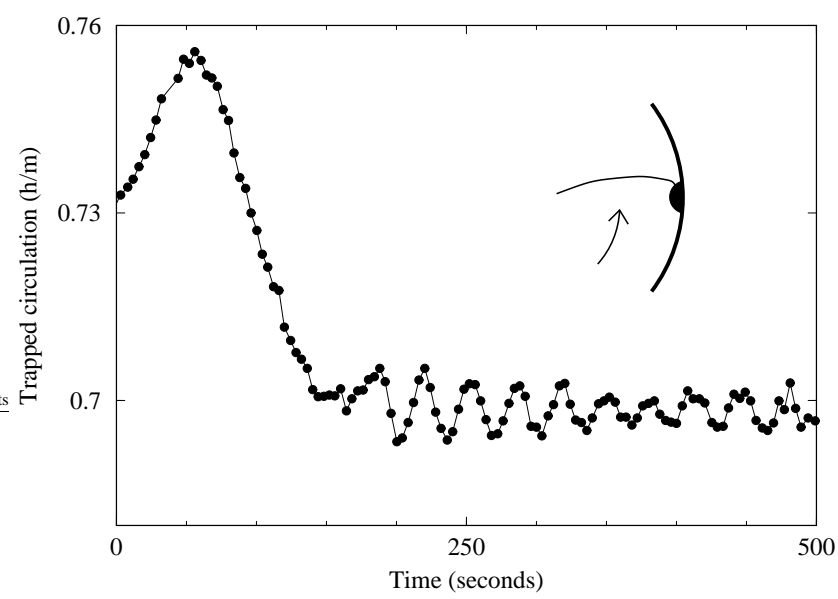

FIG. 2: Kelvin oscillations of a newly pinned vortex at $385 \mathrm{mK}$. The inset illustrates vortex behavior at a bump on the cell wall.

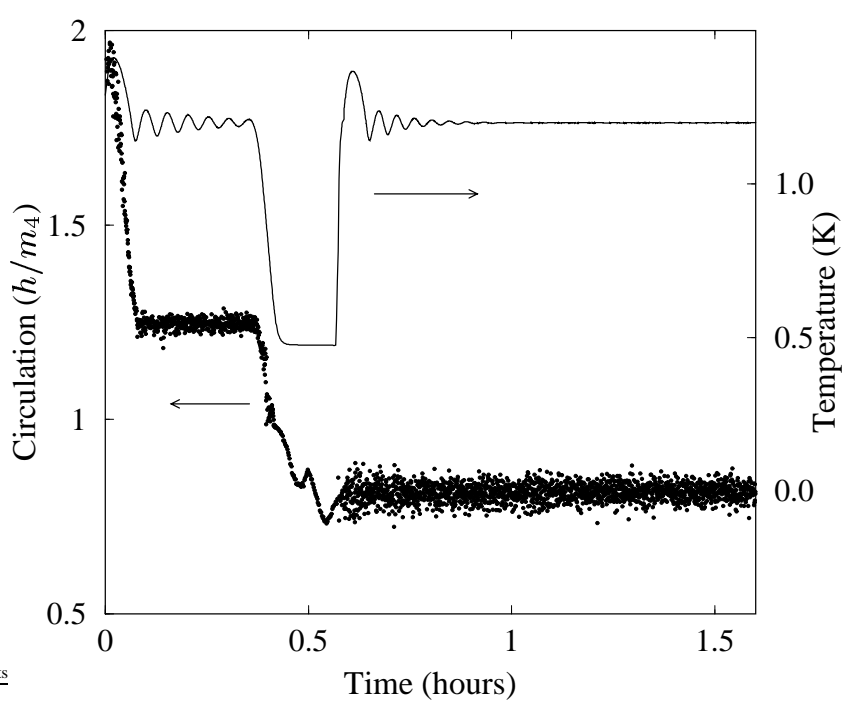

FIG. 3: Circulation (left axis) and temperature (right axis) as a function of time. At low temperatures the oscillations characteristic of vortex precession are observed, while at high temperatures neither the oscillations nor any change in the circulation level can be seen.

higher temperatures because of thermal activation. Our data suggest the opposite: depinning occurs more readily at low temperature.

Pinning events become more common as temperature increases. For cell A, we observed four events in over $40 \mathrm{~h}$ of precession measurements below $350 \mathrm{mK}$, three events in 8 hours of data between $350 \mathrm{mK}$ and $700 \mathrm{mK}$, and nine in less than one hour of vortex motion above $700 \mathrm{mK}$. Figure 3 demonstrates pinning at our highest temperatures. The cryostat was rotated immediately before the data pictured. The circulation decreases briefly, then levels off at a nonquantized value and remains there for a full 18 minutes at a temperature above $1 \mathrm{~K}$, with no sign of vortex precession. On cooling to $475 \mathrm{mK}$, the vortex dislodges and begins its characteristic spiral path through the cell. Warming above $1 \mathrm{~K}$ again produces a constant level which persists for an hour until the cryostat warms up. The increased noise at high temperature hides any Kelvin oscillations, but the absence of both dissipation and precession-induced oscillations implies that the vortex is pinned. In cell A the vortex consistently pins within minutes of raising the temperature above $700 \mathrm{mK}$. In cell $\mathrm{B}$, with its smoother walls, pinning rarely occurs at any temperature.

We next discuss the energy dissipation during vortex precession. In the low-temperature regime, where we usually observe precession without pinning, the dissipation increases sharply with increasing temperature. The inset of Figure 4 shows a precessing vortex line, with temperature abruptly changed from $340 \mathrm{mK}$ to $380 \mathrm{mK}$. When the temperature goes up, the precession continues but the background slope increases sharply. The main portion of Figure 4 shows that the decay rate rises steadily with temperature to above 500 $\mathrm{mK}$. 
The decay rate is significant because the energy dissipation comes from the same vortex-wall interaction that produces pinning. One piece of evidence is that decay rates in cell $\mathrm{B}$ are more than a factor of 5 slower than those in cell $\mathrm{A}$, as expected if cell B has smoother walls. In fact, the data in the main part of Figure 4 are from cell B, since its lower dissipation reduces the scatter in the higher temperature measurements. Cell A shows qualitatively similar behavior.

Interaction of the end of the vortex with the container wall is the only known mechanism that provides such large dissipation. Ignoring the displacement of the wire from the center of the cylinder, the energy stored per length of the trapped circulation is $u=\left(\rho_{s} \kappa^{2} / 4 \pi\right) \ln \left(R / R_{w}\right)$. Here $\rho_{s}$ is the superfluid density, $\kappa=h / m_{4}$ is the circulation quantum, $R$ is the cylinder radius, and $R_{w}$ is the wire radius. The vortex of Figure 1 detaches completely from the $5 \mathrm{~cm}$ long wire in about 30 minutes; so the average velocity $v_{z}$ is of order $3 \times 10^{-3} \mathrm{~cm} / \mathrm{s}$ and the power dissipated is $\dot{U}=u v_{z} \approx 2 \times 10^{-10} \mathrm{erg} / \mathrm{s}$.

Early experiments on vortex rings measured energy loss due to the scattering of moving vortices from normal excitations and solvated ${ }^{3} \mathrm{He}$ atoms [19]. Typical rings of radius $10^{-4} \mathrm{~cm}$, moving at $50 \mathrm{~cm} / \mathrm{s}$, lost "negligible" energy in traveling several $\mathrm{cm}$ at $280 \mathrm{mK}$ but up to $2.4 \times 10^{-10} \mathrm{erg} / \mathrm{s}$ at $650 \mathrm{mK}$. Our moving vortex has length about $0.15 \mathrm{~cm}$ and velocity about $3 \times 10^{-3} \mathrm{~cm} / \mathrm{s}$. Since dissipation is proportional to the vortex length and the square of its velocity [20], our energy loss should be more than $10^{6}$ times smaller than that of the vortex rings. Yet in fact the two are comparable at 650 $\mathrm{mK}$, and at lower temperatures our loss is larger. Thus normal excitations and ${ }^{3} \mathrm{He}$ cannot produce our observed damping.

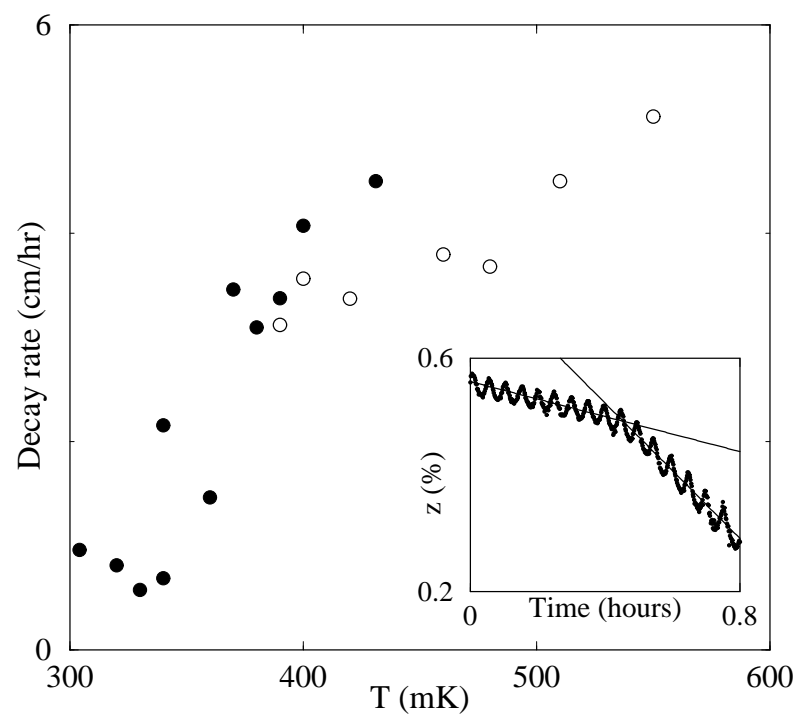

FIG. 4: Decay rate as a function of temperature (cell B). Filled circles represent decays from $\bar{\kappa}=1$ to $\bar{\kappa}=0$, while open circles are from $\bar{\kappa}=2$ to $\bar{\kappa}=1$. Inset: increase in energy dissipation rate as temperature increases from $340 \mathrm{mK}$ to $380 \mathrm{mK}$ (cell A). The lines are guides to the eye.
For vortex-boundary interactions, the only previous measurements involve abrupt changes to a cylindrical container's angular velocity [21]. As the superfluid returns to equilibrium with the cylinder, vortices move radially, their ends brushing along the top and bottom walls. For smooth walls, the dissipation is consistent with vortices scattering normal fluid entrained by the cylinder walls. For rough walls, an additional vortex-boundary force, independent of velocity, is attributed to vortex pinning [21]. The stored energy per length of a vortex creates a line tension, given by $u_{f} \approx\left(\rho_{s} \kappa^{2} / 4 \pi\right) \ln \left(R / a_{o}\right)$ for a vortex centered in a cylinder of radius $R$. Here $a_{o}=$ $1.3 \times 10^{-8} \AA$ is the radius of the free vortex core. The vortex exerts whatever force is needed to overcome the pinning, with the restriction that it cannot apply a force greater than its line tension. The resulting maximum dissipation is $\dot{U}=u_{f} 2 \pi R / T=8.8 \times 10^{-10} \mathrm{erg} / \mathrm{s}$, consistent with our observed energy loss.

Further evidence for vortex-wall dissipation is that the decay rates are comparable for $0<\bar{\kappa}<1$ and for $1<\bar{\kappa}<2$. In the latter case, the circulation about the wire changes from $\kappa=2$ to $\kappa=1$, with a $\kappa=1$ free vortex undergoing the precession. Although three times as much energy is lost in going between the $\kappa=2$ and $\kappa=1$ states as between $\kappa=1$ and $\kappa=0$, the larger velocity field increases the precession frequency by the same factor. For a velocity-independent force these two effects cancel so that $v_{z}$ is unchanged; for a velocity-dependent interaction they do not.

As previously mentioned, the dissipation is stongly temperature dependent. Yet the only explicit temperature dependence in the line tension mechanism is through the superfluid density $\rho_{s}$, which decreases monotonically with temperature. At our lowest temperature, $\rho_{s}$ essentially equals the total fluid density. Even at $1 \mathrm{~K}, \rho_{s}$ is within $0.1 \%$ of its low-temperature limit. Instead, the observed temperature dependence appears because the pinning becomes more effective as temperature increases. As the pinning force grows stronger, the force countering it from vortex line tension also grows, and with it the dissipation. Once the pinning strength exceeds the line tension, the vortex can no longer pull free, and dissipation vanishes, as shown in Figure 3 . Thus both the temperature dependence of the energy loss rate and the more direct evidence that vortex motion stops at high temperature point to an increase in pin strength with temperature.

In cell $\mathrm{A}$ the dissipation increases steadily up to $450 \mathrm{mK}$. From $450 \mathrm{mK}$ until the complete pinning begins around 700 $\mathrm{mK}$, the decay rate is too fast for us to measure accurately. The highest dissipation observed is about half the line tension limit, although the circulation disappears so quickly that the error in identifying the slope is large. In cell B, where the vortex continues to move at our highest temperatures, this maximum force is apparently never needed; and indeed the dissipation never reaches the largest values found in cell A.

Our explanation is that the wire itself excites vortex waves when we make our measurements. As the wire vibrates, typically with an initial amplitude of $10 \mu \mathrm{m}$, the end of the free vortex lying on the wire is forced to move with it. This motion excites a Kelvin wave along the free vortex. If the oscillation extends to the far end of the vortex with significant ampli- 


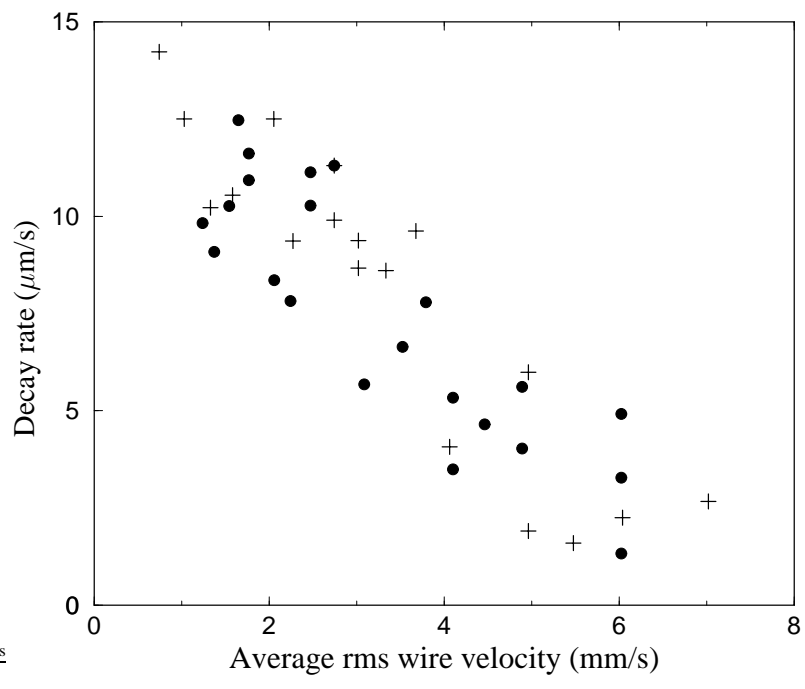

FIG. 5: Decay rate of trapped vorticity, as a function of average wire speed. $+: T=304-550 \mathrm{mK}, 9 \mathrm{~s}$ between pulses. $\bullet: T=400 \mathrm{mK}$, 4-20 $\mathrm{s}$ between pulses.

tude, it helps the vortex to wriggle free from pinning sites on the wall. The damping of the wire's motion increases sharply with temperature, making the waves less influential at higher temperatures. In this scenario, the depinning at low temperature seen in Figure 3 comes from Kelvin waves shaking the vortex free.

We have verified that motion of the wire reduces dissipation by the moving vortex. Figure 5 shows the decay rate as a function of the wire's average rms velocity $v_{a v}$. We calculate $v_{a v}$ by integrating the observed exponential decay,

$$
v_{a v}=\frac{v \tau(T)}{\Delta t}\left(1-e^{-\Delta t / \tau(T)}\right) .
$$

Here $v$ is the initial rms velocity, $\tau(T)$ the time constant of the wire's vibration, and $\Delta t$ the interval between measurements. We plot the data from Figure 4, along with data at constant temperature and varying intervals between pulses. Increasing either temperature or time between wire pulses corresponds to moving to the left in the figure. The overlay of the two sets confirms that wire motion is the primary influence on dissipation.

We suggest that the dissipation during precession comes about as the vortex encounters wall roughness that distorts it without permanently pinning it. If the vortex depins upon achieving a critical angle with the wall [14], Kelvin waves generated by the wire could help the vortex reach this angle. As the vortex leaves a temporary pin site, its shape is distorted from the usual precession configuration, giving rise to a line tension component in the direction of motion. Uninterrupted, the vortex would oscillate about the wall normal and average away the work done by vortex line tension. However, if the vortex encounters another pin site on a time scale shorter than a given mode's period, then that mode's contribution will not average away. The slowest modes, such as the oscillation of
Figure 2 with period $33 \mathrm{~s}$, thus affect the average work from line tension much more than those generated by the wire, with period near $3 \mathrm{~ms}$. At high temperatures, the wire and the associated high-frequency modes contribute less to depinning. This leads to a larger contribution from long-wavelength distortion, and more dissipation.

In conclusion, we find an increase in vortex pinning with temperature for a single vortex pinned only at one end. We show that the wire's vibration, mediated by Kelvin waves along the wire, controls vortex depinning. Our further plans include experiments with smoother and better characterized cell walls, and tests of the effects of Kelvin oscillations by exciting the wire at different frequencies between measurements. Our measurement technique allows a study of the interaction between a vortex and a single pin site, which underlies vortex phenomena in superconductors and neutron stars as well as in liquid helium.

We thank C. Olson, C. Reichhardt, and D. Thouless for helpful conversations, and UC Davis for funding.

\section{REFERENCES}

[1] L. Civale, A.D. Marwick, T.K. Worthington, M.A. Kirk, J.R. Thompson, L. Krusin-Elbaum, Y. Sun, J.R. Clem, and F. Holtzberg, "Vortex confinement by columnar defects in $\mathrm{YBa}_{2} \mathrm{Cu}_{3} \mathrm{O}_{7}$ crystals - enhanced pinning at high fields and temperatures," Phys. Rev. Lett. 67, 648 (1991).

[2] L. Civale, L. Krusin-Elbaum, J.R. Thompson, R. Wheeler, A.D. Marwick, M.A. Kirk, Y.R. Sun, F. Holtzberg, and C. Feild, "Reducing vortex motion in $\mathrm{YBa}_{2} \mathrm{Cu}_{3} 0_{7}$ crystals with splay in columnar defects," Phys. Rev. B50, 4102 (1994).

[3] L. Fruchter, A.P. Malozemoff, I.A. Campbell, J. Sanchez, M. Konczykowski, R. Griessen, and F. Holtzberg, "Low-temperature magnetic relaxation in $\mathrm{YBa}_{2} \mathrm{Cu}_{3} \mathrm{O}_{7-\delta}$-evidence for quantum tunneling of vortices," Phys. Rev. B43, 8709 (1991).

[4] D. Langlois, "Superfluidity in relativistic neutron stars," astro-ph/0008161.

[5] P.B. Jones, "Strong interaction of vortices with attractive point defects, and application to neutron star rotation", Phys. Rev. Lett. 81, 4560 (2000).

[6] C. Simon, A. Pautrat, C. Goupil, N. Lutke-Entrup, B. Placais, Y. Simon, P. Mathieu, A. Rykov, and S. Tajima, "Evidence for vortex pinning by surface irregularities in untwinned YBaCuO crystals," Physica C332, 61 (2000).

[7] R.B. Flippen, T.R. Askew, J.A. Fendrich, and C.J. van der Beek, "Surface flux pinning in $\mathrm{YBa}_{2} \mathrm{Cu}_{3} \mathrm{O}_{7-\delta}$," Phys. Rev. B52, R9882 (1995).

[8] A. Hoffmann, P. Prieto, and I.K. Schuller, "Periodic pinning with magnetic and nonmagnetic dots: the influence of size," Phys. Rev. B61, 6958 (2000).

[9] W.F. Vinen, "The detection of a single quantum of circulation in liquid helium II," Proc. Roy. Soc. London A260, 218 (1961).

[10] S.C. Whitmore and W. Zimmermann, Jr., "Observation 
of quantized circulation of superfluid helium," Phys. Rev. 166, 181 (1968).

[11] R.J. Zieve, Y. Mukharsky, J.D. Close, J.C. Davis, and R.E. Packard, "Precession of a single vortex line in superfluid ${ }^{3} \mathrm{He}$," Phys. Rev. Lett. 68, 1327 (1992).

[12] R.J. Zieve, J.D. Close, J.C. Davis, and R.E. Packard, "New experiments on the quantum of circulation in ${ }^{4}$ He," J. Low Temp. Phys. 90, 243 (1993).

[13] S.G. Hegde and W.I. Glaberson, "Pinning of superfluid vortices to surfaces," Phys. Rev. Lett. 45, 190 (1980).

[14] K.W. Schwarz, "Three-dimensional vortex dynamics in superfluid ${ }^{4} \mathrm{He}$ : Line-line and line-boundary interactions," Phys. Rev. B31, 5782 (1985).

[15] R.J. Zieve and L.A.K. Donev, "Stable vortex configurations in a cylinder," J. Low Temp. Phys. 121, 199 (2000) and cond-mat/0006078.
[16] R.J. Donnelly, Quantized Vortices in Helium II (Cambridge University Press, Cambridge, 1991), p. $28 \mathrm{ff}$.

[17] K.W. Schwarz, "Unwinding of a single quantized vortex from a wire," Phys. Rev. B47, 12030 (1993).

[18] P. Hakonen, O. Avenel, E. Varoquaux, "Evidence for single-vortex pinning and unpinning events in superfluid He-4," Phys. Rev. Lett. 81, 3451 (1998).

[19] G.W. Rayfield and F. Reif, "Quantized vortex rings in superfluid helium,” Phys. Rev. 136, A1194 (1964).

[20] C.F. Barenghi, R.J. Donnelly, and W.F. Vinen, "Friction on quantized vortices in helium II: a review," J. Low Temp. Phys. 52, 189 (1983).

[21] P.W. Adams, M. Cieplak, and W.I. Glaberson, "Spin-up problem in superfluid ${ }^{4} \mathrm{He}$," Phys. Rev. B32, 171 (1985). 